科 学 通 报

\title{
三维非线性电磁谐振腔的理论分析 *
}

\author{
周乐柱 \\ (北京大学无线电电子学系, 北京 100871)
}

\section{关链词电磁场理论、诺振腔、非线性光波导、有限元、边楼元}

具有非线性材料的光和微波器件给出了一系列新现象, 有很多潜在的应用 ${ }^{[1,2]}$, 受到人们 广泛的关注. 已有不少文章研究了波在非线性光波导中的传输, 发现其具有自聚焦和双稳的

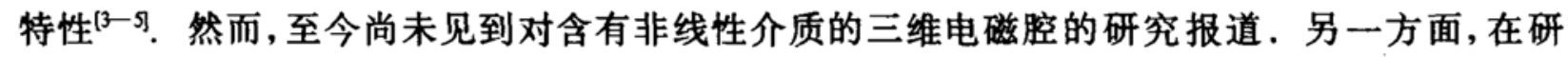
究非线性光波导的诸方法中, 最常用的是有限元法, 它突破了早期研究非线性光波传输的两 点近似 (其一为标量近似, 其二为非线性特性的平方律近似), 采用了全矢量和实际的非线性 规律的表达. 然而传统的有限元 (节点元) 具有虚模、即非物理解干扰的缺点. 为了消除虚 模干扰, 人们引人了补偿函数, 但是它的应用依赖于使用者的经验, 而且大大增加了计算量. 为了克服这一缺点, 最近几年兴起了一种新型计算方法, 它仍然基于有限元网格划分, 但它不 是用场在单元节点上的值来展开, 而是用场在单元边棱上的线积分值来展开, 因此称为边棱 元法 (Edge-element method) ${ }^{[6-8]}$. 它不仅消除了虚模干扰, 而且具有变量少、计算量小等优 点. 本文利用边棱元这一新方法来研究非线性三维谐振腔, 得到了谐振频率和模式场分布依 赖于腔总功率的结果, 这个结果预示了研制新型光波和微波器件的可能性.

\section{1 普通三维电磁问题的边棱元法}

已经证明,三维电磁问题可以用变分公式

$$
F(\boldsymbol{H})=\iiint\left\{(\nabla \times \boldsymbol{H}) \cdot \varepsilon^{-1} \cdot(\nabla \times \boldsymbol{H})-k^{2} \boldsymbol{H} \cdot \mu \cdot \boldsymbol{H}\right\} \mathrm{d} V
$$

来表达 ${ }^{[9]}$. 为了离散化 (1) 式, 将整个区域划分为有限多个小单元一一本文为小四面体. 在每 个四面体中,物质特性 $\varepsilon$ 和 $\mu$ 为常量, 任一点 $\boldsymbol{r}$ 的磁场用

$$
\boldsymbol{H}(\boldsymbol{r})=\sum_{i=1}^{6} h_{i} \boldsymbol{W}_{i}(\boldsymbol{r})
$$

来展开, 其中 $h_{i}$ 是磁场沿该四面体第 $i$ 条边棱的线积分, 即

$$
h_{i}=\int_{(i)} \boldsymbol{H} \cdot \mathrm{d} l
$$

是待求的未知变量; $\boldsymbol{W}_{i}(\boldsymbol{r})$ 是边棱元法的矢量型函数

\footnotetext{
1993-0430 收稿, 199402-03 收修改稿.

*国家数委和北京大学自然科学预研基金资助项目.
} 


$$
\boldsymbol{W}_{i}(\boldsymbol{r})=\lambda_{(i \mathrm{i})}^{(r)} \nabla \lambda_{(\mathrm{i})}^{(r)}-\lambda_{(i 2)}^{(r)} \nabla \lambda_{(i)}^{(r)},
$$

$\lambda_{(i 1)}(\boldsymbol{r})$ 和 $\lambda_{(i 2)}(\boldsymbol{r})$ 分别为第 $i$ 条边棱的两个端点 (即四边体的两个顶点) 所对应的体积坐 标一一传统节点元的标量型函数, $\nabla$ 为梯度算符. 这样

$$
\nabla \times \boldsymbol{H}=\sum_{i=1}^{6} h_{i} \nabla \times \boldsymbol{w}_{i}(\boldsymbol{r})=\sum_{i=1}^{6} 2 h_{i}\left[\nabla \lambda_{(i)}(\boldsymbol{r}) \times \nabla \lambda_{(i 2)}(\boldsymbol{r})\right] .
$$

把 (2)、(4) 和 (5) 式代人 (1) 式, 整个区域内连续的体积分就化为有限多个小单元体积分之 和, 再化为未知变量的二次多项式; 合并同类项并利用边界条件后, 令 $F$ 对所有独立的 $h$ 取极 值, 便得到代数形式的本征值方程

$$
\boldsymbol{A} \mathscr{H}=k^{2}(\boldsymbol{B} \mathscr{H}),
$$

其中 $\boldsymbol{A}$ 和 $\boldsymbol{B}$ 是总体矩阵, $\mathscr{C}$ 是包含所有独立变量的列矢量.

\section{2. 非线性三维电磁问题的迭代法}

设所考虑的区域包含非线性材料, 其非线性特性用

$$
D=\varepsilon \varepsilon_{0} E, \varepsilon=\varepsilon_{\text {lin }}+\Delta \varepsilon_{\text {sat }}\left\{1-\exp \left(-\alpha|E|^{2} / \Delta \varepsilon_{\text {sat }}\right)\right\}
$$

表示, 其中 $\varepsilon_{\text {in }}$ 是低功率 (线性)介电常数, $\Delta \varepsilon_{\text {sat }}$ 是当 $|E|$, 即总能量趋于无限时介电常数的最大 变化 (饱和值), $\alpha$ 是材料的非线性 Kerr 系数. 这是一个实际的规律, 在低功率下呈平方律, 在 高功率下趋于饱和状态 [].

原则上, (1) 式对非线性材料也成立, 但 (6) 式的推导过程应用了介质特性与场无关的假 定. 为了利用 (6) 式求解非线性问题, 提出如下迭代法:

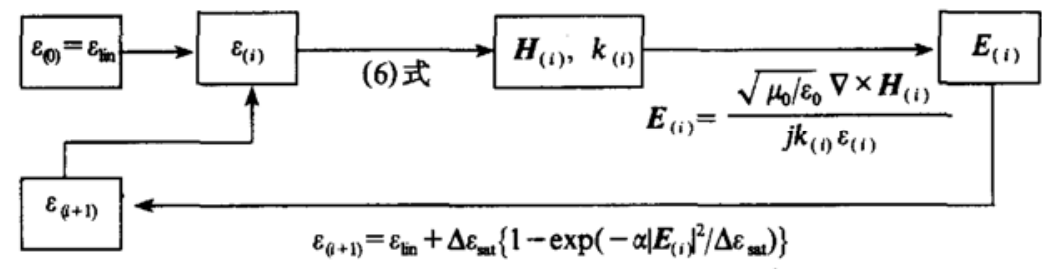

其中 $(i)$ 和 $(i+1)$ 代表迭代序号. 从 $\left.\varepsilon_{(i)}\right|_{i=0}=\varepsilon_{\text {lin }}$ 出发, 利用普通电磁问题的边棱元法和麦克斯 韦方程可求出第 $i$ 次迭代的本征值 $k_{(i)}, \omega_{(i)}$ 和场分布 $\boldsymbol{H}_{(i)}, \boldsymbol{E}_{(i)}$, 利用 (7) 式可求得下一次迭代 的介电常数值 $\varepsilon_{(i+1)}$. 迭代的每一步, 场幅值都要用所要求的总功率来标度. 迭代进行到相邻两 次本征值之差小于所给的精度为止.

\section{3 计 算 结 果}

作为例子,我们计算了一个金属壁封闭的矩形谐振腔, 其三边边长分别为 $0.4 a, a$ 和 $0.3 a$, 底部正中有一个非线性材料的六面体 (介电常量为 $\varepsilon_{2}$, 三边边长分别为 $0.15 a, 0.25 a$ 和 $0.175 a)$, 其余空间充满介电常量为 $\varepsilon_{1}$ 的线性介质. 为省计算量, 只计算其 $1 / 4$ 的结构, 并令其 内界面为理想磁壁, 其几何结构和尺寸与文献[7]的图 1 完全相同. 之所以这样选择, 是为了检验程序的可靠性. 当 $a=1 \mathrm{~cm}, \varepsilon_{1}=1, \varepsilon_{2}=16$ 时, 计算得到的谐振频率与文献 [7] 和 和脚注 1) 的完全相同, 在一定程度上证实了理论和程序的正确性.

1) Zhou Le-zhu et al., International Journal of Numerical Modelling (in press) 


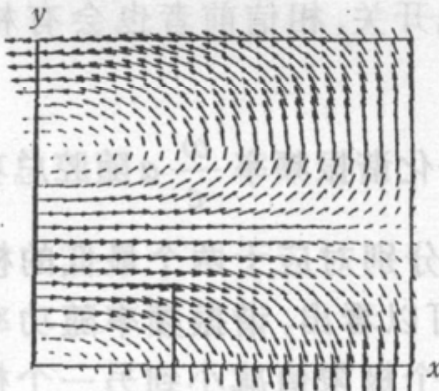

(a)

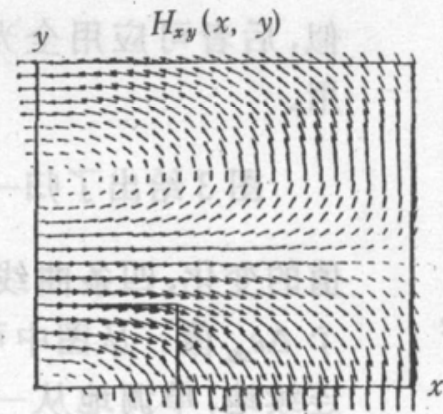

$(b-1)$

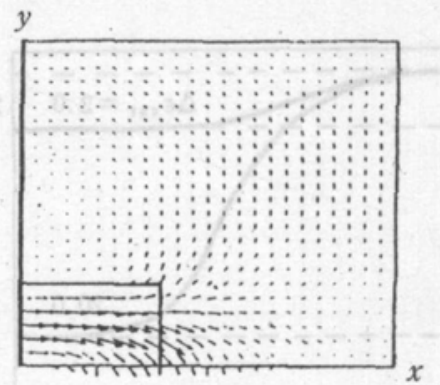

(b-2)

图 1 磁场矢量在 $x, y$ 平面 $(z=0.0875 \mathrm{a})$ 的二维矢量图

(a) 低功率, (b-1) 高功率, $\Delta \varepsilon_{\mathrm{sat}}=2.0$, (b-2) 高功率, $\Delta \varepsilon_{\mathrm{sat}}=20.0$

然后, 又选择了非线性光学中普遍采用的值 ${ }^{[s]}$

$$
\varepsilon_{1}=1.57^{2}, \varepsilon_{2}=1.55^{2}+\Delta \varepsilon_{\text {sat }}\left\{1-\exp \left(-\alpha|E|^{2} / \Delta \varepsilon_{\text {sat }}\right)\right\},
$$

其中 $\alpha=10^{-9} \mathrm{~m}^{2} / \omega$, 为便于分析和观察非线性性, $\Delta \varepsilon_{\text {sat }}=2.0$ 和 20.0 , 取了较大的值. 为使计算 程序和结果具有普遍性, 我们用腔的最大边长 $a$ 来归一化与长度有关的量, 例如谐振频率归 一化为无量纲量 $\bar{\omega}=\frac{\omega}{c} a$, 当 $a$ 改变时, 实际谐振频率 $\omega=\bar{\omega} \frac{c}{a}$ 可由计算得到的 $\bar{\omega}$ 和实际长 度 $a$ 得到. 这是电磁场数值计算中普遍采用的方法. 计算中, 四分之一的腔体积被划分为 1225 个四面体, 利用边界条件后, 总体矩阵的阶为 1575 . 迭代精度, 即最后相邻两次本征值的 相对差别小于 $10^{-3}$. 计算结果示于图 1、图 2 和图 3.

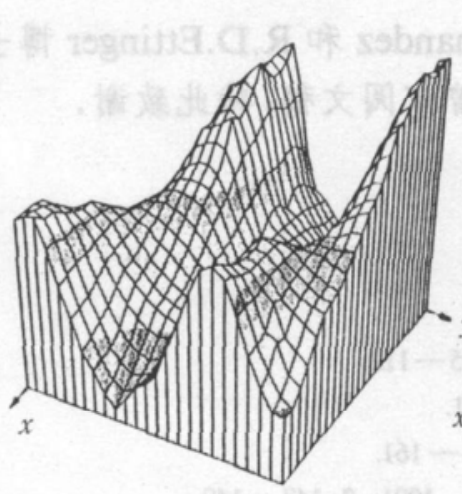

(a)

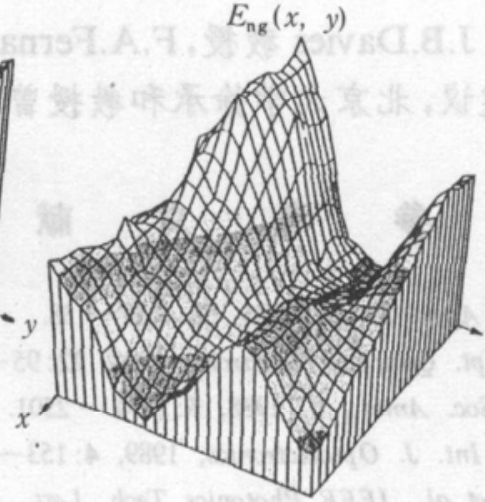

$(b-1)$

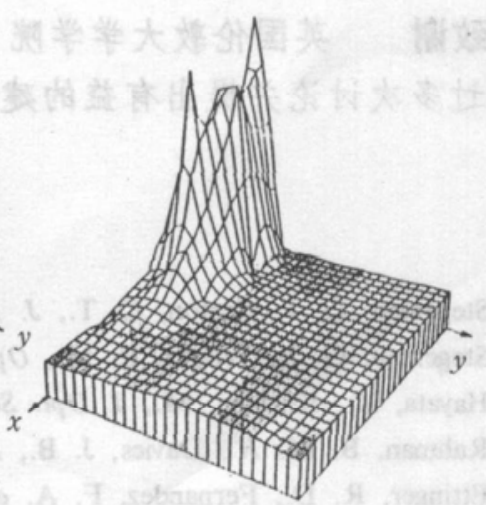

(b-2)

图 2 能量密度在 $x, y$ 平面 $(z=0.0875 a)$ 的分布

(a) 低功率, (b-1) 高功率, $\Delta \varepsilon_{\text {sat }}=2.0,(b-2)$ 高功率, $\Delta \varepsilon_{\text {set }}=20.0$

图 1 和图 2 分别给出了磁场矢量在 $z=0.0875 a$ 的横截面 (该截面平行于 $x o y$ 面并通过非 线性介质正中, 图 1 中的右下角小矩形即为非线性介质区) 内的投影和腔周期平均能量密度在 该截面的分布, 两图中的 (a) 和 (b) 分别对应于低功率和高功率. 很明显, 当腔总功率由低向 高变化时,场形和能量分布的形状大体不变, 但磁力线和能量的峰值区都向非线性介质区集 中, $\Delta \varepsilon_{\text {sat }}$ 越大, 集中的现象越明显. 这种现象与非线性光波导中场和能量的自聚焦现象相类 


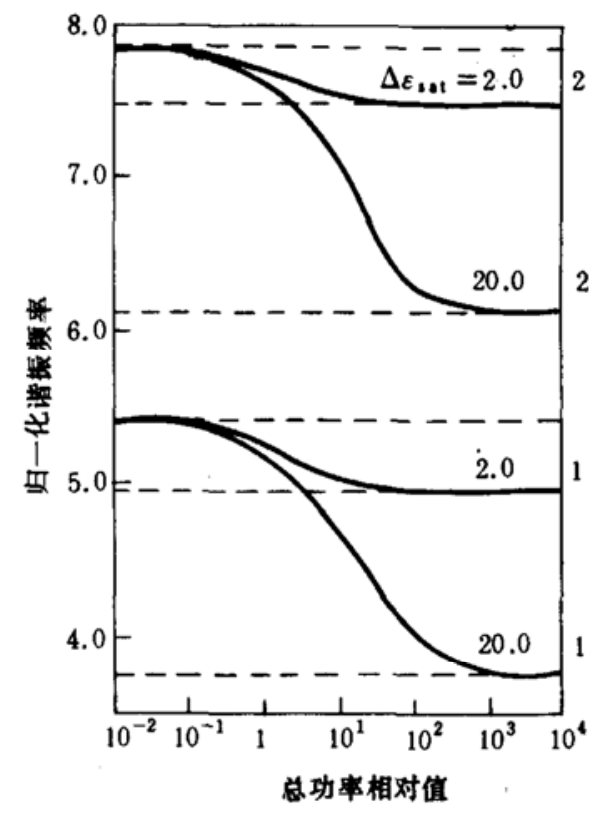

图 3 谐振频率随总功率的变化 1 为模式 1,2 为模式 2

似, 后者可应用全光开关, 相信前者也会有相应的应 用.

图 3 给出了归一化谐振频率 $\frac{\omega}{c} a$ 随腔总功率相对 值的变化, 四条曲线分别对应于两个最低的模式和两 个 $\Delta \varepsilon_{\text {sat }}$ 值. 从图中可以看出, 谐振频率随功率的增加 连续地、单调地从一个极限值减小到另一个极限值. 这种现象与非线性光波导中等效传播常数在某一功率 下会发生突变的情况不同. 谐振频率的低功率极限值 依赖于腔的几何尺寸和 $\varepsilon_{\text {lin }}$ 值, 高功率极限值还依赖于 $\Delta \varepsilon_{\text {sat }}$ 值, $\Delta \varepsilon_{\text {sat }}$ 值越大, 高功率极限值越小, 频率的可变范 围越大. 谐振频率随功率单调变化的特性可用于频率 可调谐器件.

\section{4 结 论}

本文给出了一种研究非线性三维电磁问题的方 法, 对一个具有非线性核的封闭介质谐振腔进行了实 际计算, 发现了谐振频率随总功率单调变化的特性及模式场形和能量向非线性区集中的现 象. 这些特性和现象预示了研制新的微波和光波器件(例如频率随功率可调谐的器件及其他 非线性器件)的可能性.

致谢英国伦敦大学学院 J.B.Davies 教授,F.A.Fernandez 和 R.D.Ettinger 博士曾与作 者作过多次讨论并提出有益的建议, 北京大学徐承和教授曾审阅文稿, 特此致谢.

\section{参 考献}

[1] Stegeman, G. I., Seaton, C. T., J. Appl. Phys., 1985, 58: R57- 58.

[2] Stegeman, G. I., Wright, E. M., Opt. Quantum Electron., 1990, 22:95-112.

[ 3] Hayata, K., Koshiba, M., J. Opt. Soc. Amer., B, 1988, 5:2494-2501.

[4] Rahman, B. M. A., Davies, J. B., Int. J. Optoelctronics, 1989, 4:153-161.

[5] Ettinger, R. D., Fernandez, F. A. et al., IEEE Photonics Tech. Lett., 1991, 2:147-149.

[6] Bossavit, A., Mayergoyz, I., IEEE Trans., 1989, MAG-25(2): 299-316.

[7] Bardi, J., Biro, O. et al., IEEE Trans., 1992, MAG-28(2):1142-1145.

[8] Lee, J. F., Mittra, R., IEEE Trans., 1992, MIT-40 (9): 1767-1773.

[9] Webb, J. P., Mail, G. L. et al., Proc. Inst. Elec. Eng., 1983, 3:153-159. 